\title{
Absorption of low-frequency radiation by small metal particles: a semiclassical random-phase-approximation
}

\author{
M Wilkinson $\dagger$ and B Mehlig $\ddagger$ \\ $\dagger$ Faculty of Mathematics and Computing, The Open University, Milton Keynes MK7 6AA, UK \\ $\$$ Theoretical Quantum Dynamics, Faculty of Physics, University of Freiburg, \\ Hermann-Herder-Strasse 3, D-79104 Freiburg, Germany
}

Received 12 August 2000, in final form 27 September 2000

\begin{abstract}
Calculations of low-frequency absorption of electromagnetic radiation by small conducting particles must take account of screening of the externally applied field due to polarization charges. In this paper we introduce a 'semiclassical' variant of the random-phase-approximation (RPA) method for obtaining the self-consistent field. Electric and magnetic dipole absorption are treated within a unified scheme, and we also demonstrate the equivalence of this approach to a superficially dissimilar perturbative method. The approach is more tractable than the full RPA equations, and allows us to discuss the effective potential for both diffusive and ballistic electron dynamics.
\end{abstract}

\section{Introduction}

\subsection{Motivation for examining the low-frequency response}

The interaction between electromagnetic radiation and small conducting particles has been intensively investigated. Three recent reviews [1-3] provide a survey of theoretical and experimental work in this area. Most experimental work has focused on the high-frequency response of small metal particles in an external electromagnetic field, particularly the collective excitations (Mie resonances) at frequencies comparable to the bulk plasma frequency, $\omega_{\mathrm{p}}$. Considerable success has been achieved in modelling these plasma resonances using computer programs which implement the 'random-phase approximation' (RPA) for electrons in a spherical potential (a detailed review of this approach is given in [4], and the basis of the RPA method is explained in [5]). Our paper is concerned with analysing the low-frequency regime, $\omega \ll \omega_{\mathrm{p}}$. This case is not adequately addressed by the methods used for analysing Mie resonances, and deserves a thorough treatment in its own right. The low-frequency absorption is experimentally accessible, and is of primary interest in some applications, including experiments on mesoscopic systems, and the effect of dispersed particles on planetary atmospheres. We will also show that this limit is more analytically tractable than absorption at the plasma frequency.

The low-frequency absorption can be estimated using classical electromagnetic theory. For particles which are small compared to electromagnetic length scales (wavelength and skin depth), the absorption is resolved into electric and magnetic dipole components (which are respectively proportional to $a^{3} \omega^{2} / \sigma_{0}$ and $a^{5} \omega^{2} \sigma_{0}$, where $a$ is the size and $\sigma_{0}$ the low-frequency bulk conductivity of the particles [6]). There are two fundamental reasons for which these classical estimates cannot be used without careful consideration. Firstly, the use of a bulk 
conductivity $\sigma_{0}$ implies an assumption that the electron motion is diffusive. In the case of very small systems the dimension $a$ may be smaller than the bulk mean free path of the charge carriers (this is termed the ballistic case). It has usually been assumed that the classical formulae may still be used for the absorption coefficient, with $\sigma_{0}$ replaced by an effective Drude conductivity, in which the mean free path is replaced by the system size $a$. No compelling justification of this ad hoc assumption has been given, and a calculation has been presented which implies that, in the case of two-dimensional particles, it is false [7]. Our paper discusses a semiclassical approach to the RPA which can provide a unified analysis of diffusive and ballistic electron motion.

The other reason for questioning the classical approach is that it takes no account of quantum mechanical effects. In a seminal paper, which contains many of the themes which were later developed into the field of 'mesoscopic physics', Gorkov and Eliashberg [8] proposed that the energy levels of electrons in small conducting grains should be described by randommatrix models (the properties of which are described in [9]). They proposed that there would be universal structures in the frequency dependence of the absorption coefficient, on the scale $\omega_{0}=\Delta / \hbar$, where $\Delta$ is the single-particle level spacing. When $\omega \gg \omega_{0}$, the absorption coefficient is determined by a semiclassical estimate, and is proportional to $\omega^{2}$. Gorkov and Eliashberg's calculation overestimates the coefficient of this quadratic dependence, because it neglects screening of the externally applied field by polarization charges. After nearly forty years there is still no satisfactory calculation of this coefficient for the case of ballistic electron motion. Our paper will build the theoretical basis from which such an estimate can be constructed.

It might be expected that understanding the absorption at low frequencies could be achieved from the use of the numerical implementations of the RPA method. Apart from the fact that theoretical insight is lost, this approach has limited usefulness because the implementations of the full RPA equations are confined to one special case, namely ballistic electron motion in a spherical geometry. In contrast, our semiclassical version of the RPA is much easier to apply in different geometries.

\subsection{Regimes of frequency and electron dynamics}

The problem of low-frequency absorption (meaning $\omega \ll \omega_{\mathrm{p}}$ ) clearly has many different regimes depending upon the frequency, and upon various length scales. We must clarify which cases we consider. The following frequency scales are relevant to the interaction of small metal particles with radiation. The lowest scale is given by the mean level spacing $\Delta$, i.e., $\omega_{0}=\Delta / \hbar$. In $d$ dimensions, $\omega_{0} \propto a^{-d}$, where $a$ is the characteristic size of the particle. Another frequency scale is given by the inverse of the typical time taken for an electron to traverse the particle. In systems with diffusive electron motion it is given by $\omega_{\mathrm{c}}=D / a^{2}$, where $D$ is the diffusion constant. In particles with ballistic electron motion, $\omega_{\mathrm{c}}$ is given by the inverse time of flight, $\omega_{\mathrm{c}}=v_{\mathrm{F}} / a$, where $v_{\mathrm{F}}$ is the Fermi velocity. The highest-frequency scales are the plasma frequency $\omega_{\mathrm{p}}$ and the frequency $\omega_{\mathrm{F}}=E_{\mathrm{F}} / \hbar$ derived from the Fermi energy. In two dimensions, $\omega_{\mathrm{p}} \propto a^{-1 / 2}$, whereas in three dimensions, $\omega_{\mathrm{p}}$ and $\omega_{\mathrm{F}}$ are both independent of the size of the particle (and are of comparable magnitude in the case of good metals). The frequency scales are therefore ordered as follows:

$$
\omega_{0} \ll \omega_{\mathrm{c}} \ll \omega_{\mathrm{p}}, \omega_{\mathrm{F}} .
$$

There are six relevant length scales in the problem, namely the wavelength $\lambda$ of the external radiation, the linear dimension $a$ of the particle, the skin depth $\lambda_{s}$, the Thomas-Fermi screening length $\lambda_{\mathrm{TF}}$, the Fermi wavelength $\lambda_{\mathrm{F}}$ and the mean free path $l$. In the following it will be 
assumed that

$$
\lambda, \lambda_{\mathrm{s}} \gg a \gg \lambda_{\mathrm{TF}} \sim \lambda_{\mathrm{F}} .
$$

Both diffusive $(l \ll a)$ and ballistic $(l \gg a)$ dynamics will be discussed in the present paper.

It is also natural to consider whether the electron dynamics influences the absorption properties of small conducting particles. One expects, in particular, that the low-frequency absorption (on frequency scales of the order of the mean time of flight) will depend sensitively on the nature of the classical dynamics. In recent years it has become possible to fabricate very clean two-dimensional mesoscopic devices exhibiting ballistic electron motion. The Fermi energy in semiconductors can be small, which implies that the Fermi wavelength may be larger than the surface roughness. In this case reflection of an electron colliding with the edge of the sample is specular. (For a recent review including experimental work, see [10].) The classical dynamics of the electron may therefore be that of a classical billiard, which may exhibit integrable, chaotic or mixed dynamics depending upon the shape of the boundary. It is therefore possible, in principle, to observe differences between ergodic and integrable electron dynamics in the response of two-dimensional particles.

\subsection{Contributions of this paper and relation to earlier work}

This paper is a development from earlier work which has applied semiclassical techniques to estimate the absorption of radiation. References [11] and [7] investigated the absorption by particles with ballistic electron motion, in spherical and disc-shaped particles respectively. These works used a Thomas-Fermi theory for the effective potential. This approach led to a number of new predictions concerning the low-frequency absorption in the ballistic case:

(a) In the two-dimensional case (conducting discs), it was found that the absorption coefficient increases linearly with frequency, as opposed to a quadratic increase as predicted by the conventional modified Mie theory (in which the system size is used as the scattering length in the Drude conductivity).

(b) A series of resonances was predicted, the lowest corresponding to synchrotron acceleration of the electrons.

(c) In the case of spherical particles, a mechanism was proposed which creates enhanced absorption when the surface of the particle is rough.

Despite this progress, several important questions remain unanswered to date. Later studies $[12,13]$ have shown that in diffusive systems, absorption at frequencies $\omega$ in the range $\omega_{\mathrm{c}} \ll \omega \ll \omega_{\mathrm{p}}$ is caused by a 'dynamic potential'. (In the diffusive case the absorption coefficient was shown to be exactly the same as the prediction of the classical theory described in [6].) This additional dynamic potential was not considered in [11, 7] and it is necessary to study its effect in systems with ballistic electron dynamics. We will argue that the effect of including this term leads to a quantitative difference in the results, but not to a qualitative difference.

Also, the approach used in [7] and [11-13] is based on perturbation theory and a semiclassical approximation to quantum mechanical matrix elements, and appears to be very different from the commonly used RPA described in [5]. The features described in [11, 7] have not been discussed within the quantum mechanical RPA approach in [5] and it is thus of great interest to determine to what extent the two approaches are related. We will show that the RPA and perturbative approaches are equivalent.

Within a semiclassical framework, electric and magnetic dipole absorption have been treated using different approximation schemes. The electric dipole absorption was discussed for various different situations in [7, 11-13], and the magnetic dipole absorption case was 
discussed in [14]. In this paper we give a unified approach to electric and magnetic dipole absorption.

The present paper is organized as follows. In section 2 we develop a general scheme giving a unified approach to electric and magnetic dipole absorption. Most treatments of the electric dipole absorption coefficient are based upon calculating the imaginary part of the polarizability. References [7,11-14] used an alternative approach, namely treating the effective potential as a time-dependent perturbation. In section 3 we show that these apparently dissimilar approaches are equivalent. Section 4 describes the form of the non-local polarizability in various cases, and section 5 discusses the 'semiclassical' method for determining the self-consistent field. We show that the Thomas-Fermi potential used in [11,7], which treated electric dipole absorption ballistic systems, is not a good approximation when $\omega \gg \omega_{\mathrm{c}}$. Section 6 summarizes the results, and presents an argument indicating that the results of $[11,7]$ are nevertheless qualitatively correct. A thorough analysis of this question is deferred to a later publication [15].

\section{Calculation of the absorption coefficient}

This section will discuss the general principles underlying the calculation of the electromagnetic response.

\subsection{Formulation of the problem}

An electromagnetic wave induces currents which result in both electric and magnetic polarization of a conducting particle. In what follows we will only consider linear effects (where the polarization is proportional to the applied field), and the externally applied field will be assumed to be uniform over the dimension of the particle. We will only be concerned with the coefficients relating dipolar moments to the externally applied field: higher moments will not be considered. The electric dipole $\boldsymbol{d}$ and magnetic dipole $\boldsymbol{m}$ of a single particle are given by

$$
\boldsymbol{d}=\tilde{\alpha} \boldsymbol{E}_{\mathrm{ext}} \quad \boldsymbol{m}=\tilde{\beta} \boldsymbol{B}_{\mathrm{ext}}+\tilde{\gamma} \boldsymbol{E}_{\mathrm{ext}}
$$

where $\boldsymbol{E}_{\mathrm{ext}}$ and $\boldsymbol{B}_{\mathrm{ext}}$ are the externally applied electric and magnetic fields, $\tilde{\alpha}$ and $\tilde{\beta}$ are the electric and magnetic susceptibility tensors of the particle. The cross-susceptibility $\tilde{\gamma}$ is not usually included. It is absent for spherical particles and some other symmetric geometries, and when it is non-zero it vanishes in the low-frequency limit. Another cross-susceptibility, relating the electric dipole moment to the magnetic field, might be included in (2.1). In section 5.1 we demonstrate that (within our semiclassical approximation scheme) there is no charge density generated by the action of the magnetic field, and that this additional susceptibility is therefore absent. We will ultimately give a general treatment, showing that although the coefficient $\tilde{\gamma}$ may be non-zero, this cross-susceptibility makes no contribution to the absorption. For simplicity the cross-term will be dropped in the remainder of this introductory section.

The externally applied fields are assumed to be multiplied by a factor of the form $\exp (-\mathrm{i} \omega t)$, and the polarizability tensors are understood to be functions of $\omega$ with complexvalued components, because there may be a phase shift between the applied field and the response. For example, the actual value of the dipole moment at time $t$ is taken to be $\boldsymbol{d}(t)=\operatorname{Re}[\boldsymbol{d} \exp (-\mathrm{i} \omega t)]$

The polarizations $\boldsymbol{d}$ and $\boldsymbol{m}$ are detectable at a macroscopic level in various ways: they alter the dielectric constant and magnetic permeability of the medium in which the particles are dispersed, and they may also be detected by observing scattering and absorption of radiation. The polarizability determines two processes which result in the attenuation of radiation, namely 
scattering and absorption of energy. Both of these processes can be characterized conveniently at the microscopic level by calculating the rate of loss of energy from the incident beam due to interaction with a single particle: this will be denoted by $\overline{\mathrm{d} E / \mathrm{d} t}$ (where $\bar{X}$ denotes the time average of $X(t))$. The two measures most commonly used to quantify these processes are the cross section per particle $\mathcal{S}$ and the attenuation coefficient $\gamma$. To relate the energy loss to the cross section, note that the energy density in an electromagnetic wave is $\frac{1}{2} \epsilon_{0} \boldsymbol{E}^{2}$ : the cross section is therefore

$$
\mathcal{S}=\frac{2}{\epsilon_{0} c \boldsymbol{E}^{2}} \frac{\overline{\mathrm{d} E}}{\mathrm{~d} t}
$$

The attenuation coefficient $\gamma$ is defined by the expression $I=I_{0} \exp (-\gamma z)$, where $I$ is the intensity at distance $z$ along the beam. The attenuation coefficient is given by $\gamma=\mathcal{N S}$, where $\mathcal{N}$ is the particle density.

At low frequencies the real part of the polarizability approaches a constant (and the imaginary part approaches zero). It follows that at sufficiently low frequencies the scattering cross section scales as $\omega^{4}$. It will be shown that the absorption cross section typically scales as $\omega^{2}$, implying that absorption is expected to be the dominant process at low frequencies.

The absorption of radiation can be related to the imaginary parts of the polarizability tensors: we will give a careful explanation of this. Electron spin is not significant in this context, and the full Hamiltonian for the electrons is taken to be

$H=\sum_{i=1}^{N} \frac{1}{2 m}\left[\boldsymbol{p}_{i}+e \boldsymbol{A}_{\mathrm{ext}}\left(\boldsymbol{r}_{i}, t\right)\right]^{2}+V\left(\boldsymbol{r}_{i}\right)+\phi_{\mathrm{ext}}\left(\boldsymbol{r}_{i}, t\right)+\frac{1}{2} \sum_{i=1}^{N} \sum_{\substack{j=1 \\ j \neq i}}^{N} \frac{e^{2}}{4 \pi \epsilon_{0}\left|\boldsymbol{r}_{i}-\boldsymbol{r}_{j}\right|}$.

(Throughout $e$ represents the magnitude of the electron charge, and $\phi$ is the potential energy of an electron.) The externally applied electric and magnetic fields are considered to be spatially uniform, since the particle is small compared to the wavelength of the radiation. We therefore ignore the spatial dependence of the electric and magnetic fields, and write

$$
\phi_{\text {ext }}(\boldsymbol{r}, t)=e \boldsymbol{r} \cdot \boldsymbol{E}_{\text {ext }} \quad \nabla \wedge \boldsymbol{A}_{\text {ext }}(\boldsymbol{r}, t)=\boldsymbol{B}_{\text {ext }}(t) .
$$

In the case where the circularly symmetric gauge

$$
\boldsymbol{A}_{\text {ext }}=\boldsymbol{A}_{\text {rot }}(\boldsymbol{r}, t)=\frac{1}{2} \boldsymbol{B}_{\text {ext }}(t) \wedge \boldsymbol{r}
$$

is used, the full Hamiltonian contains terms coupling the system to the electric and magnetic fields, of the following form:

$$
\begin{aligned}
& \hat{H}(t)=\hat{H}_{0}+e \hat{\boldsymbol{X}} \cdot \boldsymbol{E}_{\mathrm{ext}}(t)+\frac{e}{2 m} \hat{\boldsymbol{L}} \cdot \boldsymbol{B}_{\mathrm{ext}}(t)+\mathrm{O}\left(\boldsymbol{B}_{\mathrm{ext}}^{2}\right) \\
& \hat{\boldsymbol{X}}=\sum_{i=1}^{N} \hat{\boldsymbol{r}}_{i} \quad \hat{\boldsymbol{L}}=\sum_{i=1}^{N} \boldsymbol{r}_{i} \wedge \boldsymbol{p}_{i}
\end{aligned}
$$

where $\hat{\boldsymbol{X}}$ and $\hat{\boldsymbol{L}}$ are the total dipole operator and total angular momentum operators, and $\hat{H}_{0}$ is independent of time.

To facilitate the calculations we will consider ensemble averages of quantities: if the electron motion is ergodic, this is the microcanonical average, and in general the ensemble is defined by the region of phase space explored by the dynamics. Angle brackets will be used for the appropriate ensemble average. For a general choice of gauge the instananeous rate of absorption is then

$$
\left\langle\frac{\mathrm{d} E}{\mathrm{~d} t}\right\rangle=\left\langle\frac{\partial H}{\partial t}\right\rangle=-e\left\langle\sum_{i=1}^{N} \boldsymbol{v}_{i}(t) \cdot\left[\boldsymbol{E}_{\mathrm{ext}}(t)+\boldsymbol{E}_{\text {ind }}(t)\right]\right\rangle=\int \mathrm{d} \boldsymbol{r} \boldsymbol{j}(\boldsymbol{r}, t) \cdot\left[\boldsymbol{E}_{\text {ext }}(t)+\boldsymbol{E}_{\text {ind }}(\boldsymbol{r}, t)\right]
$$


where $\boldsymbol{v}_{i}$ is the velocity of the $i$ th electron, $\boldsymbol{E}_{\text {ind }}=\mathrm{i} \omega \boldsymbol{A}_{\text {ext }}$ is the electric field induced by the varying magnetic field and

$$
\boldsymbol{j}(\boldsymbol{r}, t)=-e\left\langle\sum_{i=1}^{N} \boldsymbol{v}_{i}(t) \delta\left[\boldsymbol{r}-\boldsymbol{r}_{i}(t)\right]\right\rangle
$$

is the current density within the particle. In the special case where the circularly symmetric gauge is used this reduces to

$$
\left\langle\frac{\mathrm{d} E}{\mathrm{~d} t}\right\rangle=\left\langle\frac{\partial H}{\partial t}\right\rangle=e\langle\hat{\boldsymbol{X}}\rangle \cdot \dot{\boldsymbol{E}}_{\text {ext }}(t)+\frac{e}{2 m}\langle\hat{\boldsymbol{L}}\rangle \cdot \dot{\boldsymbol{B}}_{\text {ext }}(t)
$$

where $\langle\hat{\boldsymbol{X}}\rangle$ and $\langle\hat{\boldsymbol{L}}\rangle$ are suitable averages of the centre-of-mass and angular momentum operators. It is impractical to calculate these averages from the full Hamiltonian (2.3), and in the next subsection it will be shown how they may be estimated using an effective Hamiltonian, containing effective fields $\boldsymbol{A}_{\text {eff }}$ and $\phi_{\text {eff }}$. At this stage we will only assume that these averages are proportional to the applied fields. These quantities $\langle\hat{\boldsymbol{X}}\rangle$ and $\langle\hat{\boldsymbol{L}}\rangle$ are related to the electric and magnetic dipole moments $\boldsymbol{d}$ and $\boldsymbol{m}$ :

$$
\boldsymbol{d}=-e\langle\hat{\boldsymbol{X}}\rangle \quad \boldsymbol{m}=-\frac{e}{m}\langle\hat{\boldsymbol{L}}\rangle .
$$

The rate of absorption is obtained by substituting for the time dependence of a monochromatic field using (2.1), and ignoring the cross-term:

$$
\begin{aligned}
\left\langle\frac{\mathrm{d} E}{\mathrm{~d} t}\right\rangle=\operatorname{Re}[ & \boldsymbol{d} \exp (-\mathrm{i} \omega t)] \cdot \operatorname{Re}\left[\mathrm{i} \omega \boldsymbol{E}_{\operatorname{ext}} \exp (-\mathrm{i} \omega t)\right] \\
& +\frac{1}{2} \operatorname{Re}[\boldsymbol{m} \exp (-\mathrm{i} \omega t)] \cdot \operatorname{Re}\left[\mathrm{i} \omega \boldsymbol{B}_{\mathrm{ext}} \exp (-\mathrm{i} \omega t)\right] .
\end{aligned}
$$

Averaging over time gives the general form for the rate of absorption:

$$
\left\langle\overline{\frac{\mathrm{d} E}{\mathrm{~d} t}}\right\rangle=\frac{1}{4} \omega \boldsymbol{E}_{\mathrm{ext}}^{*}\left(\tilde{\alpha}-\tilde{\alpha}^{*}\right) \boldsymbol{E}_{\mathrm{ext}}+\frac{1}{8} \omega \boldsymbol{B}_{\mathrm{ext}}^{*}\left(\tilde{\beta}-\tilde{\beta}^{*}\right) \boldsymbol{B}_{\mathrm{ext}} .
$$

In the case where the polarizability tensor is isotropic, and the radiation field is plane polarized, this expression becomes

$$
\left\langle\overline{\frac{\mathrm{d} E}{\mathrm{~d} t}}\right\rangle=\frac{1}{2} \omega \operatorname{Im}\left[\alpha_{i i}(\omega)\right]\left|\boldsymbol{E}_{\text {ext }}\right|^{2}+\frac{1}{4} \omega \operatorname{Im}\left[\beta_{i i}(\omega)\right]\left|\boldsymbol{B}_{\text {ext }}\right|^{2} .
$$

We note that under the assumptions listed above, the absorption is expressed as the sum of two terms, which are naturally referred to as the electric and magnetic dipole absorption. Our final result will not neglect the magnetic dipole moment which may be induced by the electric field, but we will show that within the framework of our self-consistent approximation scheme the cross-term in (2.1) makes no contribution to the absorption. The energy absorbed does not accumulate in the electronic system: most of it is eventually transformed into heat by interaction with phonons.

\subsection{Self-consistent fields}

The Hamiltonian will be approximated by an effective Hamiltonian, in which the electrons move independently. The direct interaction with the magnetic field via electron spin can also be neglected, and the effective Hamiltonian is of the form

$$
\hat{H}_{\mathrm{eff}}=\sum_{i=1}^{N} \frac{1}{2 m}\left[\hat{\boldsymbol{p}}_{i}+e \boldsymbol{A}_{\mathrm{eff}}\left(\boldsymbol{r}_{i}, t\right)\right]^{2}+V_{\mathrm{eff}}\left(\boldsymbol{r}_{i}\right)+\phi_{\mathrm{eff}}\left(\boldsymbol{r}_{i}, t\right) .
$$


The response of the system is determined by the interaction of the electrons with the electric field inside the particle, which is described by the effective potentials $\boldsymbol{A}_{\text {eff }}$ and $\phi_{\text {eff }}$. The effective potentials are themselves determined by the distribution of charge within the particle. The external magnetic field is also augmented by an induced magnetic field which is produced by the action of the currents which flow in order to establish the electric polarization. Provided the particle is sufficiently small, the induced magnetic field can be neglected, and our selfconsistent theory will yield an equation for the electric field $\boldsymbol{E}(\boldsymbol{r}, \omega)$ within the particle, which is related to the time-dependent electric field as follows:

$$
\boldsymbol{E}(\boldsymbol{r}, \omega)=\int_{-\infty}^{\infty} \mathrm{d} t \boldsymbol{E}(\boldsymbol{r}, t) \exp (-\mathrm{i} \omega t)
$$

This field satisfies the Maxwell equations

$$
\boldsymbol{\nabla} \cdot \boldsymbol{E}=\frac{\rho}{\epsilon_{0}} \quad \nabla \wedge \boldsymbol{E}=-\mathrm{i} \omega \boldsymbol{B}_{\mathrm{ext}} .
$$

The electric field produced by induction when the external electric field is zero will be denoted $\boldsymbol{E}_{\text {ind. }}$. The total effective electric field is

$$
\boldsymbol{E}(\boldsymbol{r}, \omega)=\boldsymbol{E}_{\mathrm{ext}}(\boldsymbol{r}, \omega)+\boldsymbol{E}_{\mathrm{ind}}(\boldsymbol{r}, \omega)+\frac{1}{e} \nabla \phi_{\mathrm{pol}}(\boldsymbol{r}, \omega)
$$

The uniform external electric field satisfies $\boldsymbol{\nabla} \cdot \boldsymbol{E}_{\text {ext }}=0$, and can be derived from an external potential:

$$
\boldsymbol{E}_{\mathrm{ext}}(\boldsymbol{r}, \omega)=\frac{1}{e} \nabla \phi_{\mathrm{ext}}(\boldsymbol{r}, \omega) .
$$

The potential $\phi_{\text {pol }}$ results from polarization of the particle due to the external electric field, and is given by

$$
\phi_{\mathrm{pol}}(\boldsymbol{r}, \omega)=\frac{-e}{4 \pi \epsilon_{0}} \int \mathrm{d} \boldsymbol{r}^{\prime} \frac{\rho_{\mathrm{pol}}\left(\boldsymbol{r}^{\prime}, \omega\right)}{\left|\boldsymbol{r}-\boldsymbol{r}^{\prime}\right|}
$$

where $\rho_{\text {pol }}(\boldsymbol{r}, \omega)$ is the charge density resulting from polarization induced by the external electric field, but excluding any polarization which may result from the induction field $\boldsymbol{E}_{\text {ind }}$. It will be convenient to express (2.17) using the notation

$$
\left.\left.\left.\mid \boldsymbol{E})=\frac{1}{e} \nabla \mid \phi_{\mathrm{ext}}\right)+\mid \boldsymbol{E}_{\text {ind }}\right)+\frac{1}{e} \nabla \hat{U} \mid \rho_{\mathrm{pol}}\right)
$$

where $\hat{U}$ is an operator defined by (2.19), acting on the 'field vector' $\left.\mid \rho_{\text {pol }}\right)$. The dependence upon frequency will usually be shown explicitly for operators, but not for field vectors.

The current density $\boldsymbol{j}(\boldsymbol{r}, \omega)$ flowing in the sample to build up the charge density $\rho(\boldsymbol{r}, \omega)$ may be assumed to be linearly related to the electric field $\boldsymbol{E}(\boldsymbol{r}, \omega)$ in the sample:

$$
\boldsymbol{j}(\boldsymbol{r}, \omega)=\int \mathrm{d} \boldsymbol{r}^{\prime} \tilde{\Sigma}\left(\boldsymbol{r}, \boldsymbol{r}^{\prime} ; \omega\right) \boldsymbol{E}\left(\boldsymbol{r}^{\prime}, \omega\right) .
$$

In condensed notation, we write

$$
\mid \boldsymbol{j})=\widehat{\Sigma}(\omega) \mid \boldsymbol{E})
$$

where $\widehat{\Sigma}(\omega)$ is the conductivity operator. The non-local conductivity tensor $\tilde{\Sigma}\left(\boldsymbol{r}, \boldsymbol{r}^{\prime}, \omega\right)$ is related to the non-local polarizability operator $\Pi\left(\boldsymbol{r}, \boldsymbol{r}^{\prime}, \omega\right)$, which gives the charge density induced by a potential $\phi(r)$ : we write

$$
\rho(\boldsymbol{r}, \omega)=\int \mathrm{d} \boldsymbol{r}^{\prime} \Pi\left(\boldsymbol{r}, \boldsymbol{r}^{\prime} ; \omega\right) \phi\left(\boldsymbol{r}^{\prime}, \omega\right)
$$


or in condensed notation

$$
\mid \rho)=\hat{\Pi}(\omega) \mid \phi) .
$$

The polarizability operator $\hat{\Pi}(\omega)$ can be related to a non-local conductivity tensor $\widehat{\Sigma}(\omega)$ by a continuity relation. Using (2.23) and applying the continuity equation, we find (with summation over repeated indices implied)

$$
0=\int \mathrm{d} \boldsymbol{r}^{\prime}\left[\mathrm{i} e \omega \Pi\left(\boldsymbol{r}, \boldsymbol{r}^{\prime} ; \omega\right) \phi\left(\boldsymbol{r}^{\prime}\right)+\nabla_{i} \Sigma_{i j}\left(\boldsymbol{r}, \boldsymbol{r}^{\prime} ; \omega\right) \nabla_{j}^{\prime} \phi\left(\boldsymbol{r}^{\prime}\right)\right]
$$

assuming that the normal component of $\widehat{\Sigma}(\omega)$ vanishes on the surface. Upon integration by parts, after noting that the resulting equation is valid for any field $\phi(r, \omega)$, we find ie $\omega \Pi\left(\boldsymbol{r}, \boldsymbol{r}^{\prime} ; \omega\right)-\partial_{r_{i}} \partial_{r_{j}^{\prime}} \Sigma_{i j}\left(\boldsymbol{r}, \boldsymbol{r}^{\prime} ; \omega\right)=0$. This may be expressed in compact notation in the form

$$
\mathrm{i} e \omega \hat{\Pi}(\omega)=+\vec{\nabla} \widehat{\Sigma}(\omega) \overleftarrow{\nabla}
$$

The quantities $\hat{\Pi}(\omega)$ and $\widehat{\Sigma}(\omega)$ enable (2.20) to be expressed in terms of the electric field alone, yielding a self-consistent equation. In pursuit of this, we write

$$
\left.\left.\mid \boldsymbol{E})=\frac{1}{e} \nabla \mid \phi_{\mathrm{eff}}\right)+\mid \boldsymbol{E}_{\text {ind }}\right)
$$

where $\phi_{\text {eff }}(\boldsymbol{r}, \omega)$ is an effective potential. We consider the solutions for the field $\boldsymbol{E}_{\text {ind }}$ and the potential $\phi_{\text {eff }}$ separately. The charge induced by the field $\boldsymbol{E}_{\text {ind }}$ is

$$
\left.\left.\mid \rho_{\text {ind }}\right)=\frac{1}{\mathrm{i} \omega} \nabla \widehat{\Sigma}(\omega) \mid \boldsymbol{E}_{\text {ind }}\right) .
$$

Applying the first of the Maxwell equations (2.16) and using (2.28) gives

$$
\left.\left.\nabla \cdot\left[\mid \boldsymbol{E}_{\text {ind }}\right)-\frac{\mathrm{i}}{\epsilon_{0} \omega} \widehat{\Sigma}(\omega) \mid \boldsymbol{E}_{\text {ind }}\right)\right]=0
$$

which is the self-consistent equation which must be solved for the field $\boldsymbol{E}_{\text {ind }}$. For the effective potential, combining (2.20) with (2.24) and (2.27), we find

$$
\left.\left.\left.\mid \phi_{\text {eff }}\right)=\mid \phi_{\text {ext }}\right)+\hat{U} \hat{\Pi}(\omega) \mid \phi_{\text {eff }}\right) .
$$

This self-consistent equation is sometimes referred to as the 'random-phase approximation' [5]. Equations (2.29) and (2.30) must be solved for the self-consistent fields. We will consider semiclassical methods for solving them in section 5 .

\subsection{The rate of energy absorption}

The rate of energy absorption is given by (2.7). Averaging over time gives

$$
\left\langle\overline{\frac{\mathrm{d} E}{\mathrm{~d} t}}\right\rangle=\frac{1}{2} \operatorname{Re} \int \mathrm{d} \boldsymbol{r} j^{*}(\boldsymbol{r}, \omega) \cdot\left[\boldsymbol{E}_{\mathrm{ext}}+\boldsymbol{E}_{\text {ind }}(\boldsymbol{r}, \omega)\right] .
$$

In condensed notation this will be written, by analogy with Dirac notation, as

$$
\left\langle\overline{\frac{\mathrm{d} E}{\mathrm{~d} t}}\right\rangle=\frac{1}{2} \operatorname{Re}\left(\boldsymbol{j} \mid \boldsymbol{E}_{\text {ext }}\right)+\frac{1}{2} \operatorname{Re}\left(\boldsymbol{j} \mid \boldsymbol{E}_{\text {ind }}\right) .
$$

Using (2.20),

$\left\langle\overline{\left.\frac{\mathrm{d} E}{\mathrm{~d} t}\right\rangle}\right\rangle=\frac{1}{2} \operatorname{Re}(\boldsymbol{j} \mid \boldsymbol{E})-\frac{1}{2 e} \operatorname{Re}\left(\boldsymbol{j}|\nabla \hat{U}| \rho_{\mathrm{pol}}\right)=\frac{1}{2} \operatorname{Re}(\boldsymbol{j} \mid \boldsymbol{E})-\frac{1}{2 e} \operatorname{Re}\left[\mathrm{i} \omega\left(\rho_{\mathrm{pol}}|\hat{U}| \rho_{\mathrm{pol}}\right)\right]$ 
where $\left.\left.\mid \rho_{\text {pol }}\right)=\hat{\Pi} \mid \phi_{\text {eff }}\right)$; the final equality follows from an integration by parts, and use of the continuity equation. Using the fact that $\hat{U}$ is self-adjoint, we obtain a very simple expression for the absorption:

$$
\left\langle\overline{\frac{\mathrm{d} E}{\mathrm{~d} t}}\right\rangle=\frac{1}{2} \operatorname{Re}(\boldsymbol{j} \mid \boldsymbol{E}) .
$$

Using the continuity equation and (2.20), (2.26) and (2.27),

$(\boldsymbol{j} \mid \boldsymbol{E})=\frac{\mathrm{i} \omega}{e}\left(\phi_{\mathrm{eff}}\left|\hat{\Pi}^{+}(\omega)\right| \phi_{\mathrm{eff}}\right)+\left(\boldsymbol{E}_{\text {ind }}\left|\widehat{\Sigma}^{+}(\omega)\right| \boldsymbol{E}_{\text {ind }}\right)+\frac{1}{e}\left(\boldsymbol{j}_{\mathrm{ind}} \mid \nabla \phi_{\mathrm{eff}}\right)+\frac{1}{e}\left(\nabla \phi_{\mathrm{eff}} \mid \boldsymbol{j}_{\text {ind }}\right)$.

where $\left.\left.\mid j_{\text {ind }}\right)=\widehat{\Sigma} \mid \boldsymbol{E}_{\text {ind }}\right)$, and where the adjoint $\hat{A}^{+}$of an operator $\hat{A}$ satisfies $\left(a\left|\hat{A}^{+}\right| b\right)=$ $(a|\hat{A}| b)^{*}$ for any states $\left.\left.\mid a\right), \mid b\right)$. Using an integration by parts and the continuity equation, the last two terms may be rearranged as follows:

$$
\left(\nabla \phi_{\text {eff }} \mid j_{\text {ind }}\right)=-\left(\phi_{\text {eff }} \mid \nabla \cdot j_{\text {ind }}\right)=\mathrm{i} \omega\left(\phi_{\text {eff }} \mid \rho_{\text {ind }}\right)
$$

where $\left(\rho_{\text {ind }}\right)$ is the charge density induced by the magnetic field. In section 5.1 it will be shown that $\left(\rho_{\text {ind }}\right)$ vanishes in our semiclassical approximation. This gives our final expression for the absorption:

$$
\left\langle\overline{\frac{\mathrm{d} E}{\mathrm{~d} t}}\right\rangle=\frac{\omega}{2 e} \omega \operatorname{Im}\left(\phi_{\mathrm{eff}}|\hat{\Pi}(\omega)| \phi_{\text {eff }}\right)+\frac{1}{2} \operatorname{Re}\left(\boldsymbol{E}_{\text {ind }}|\widehat{\Sigma}(\omega)| \boldsymbol{E}_{\text {ind }}\right) .
$$

These are two independent contributions to the rate of absorption, depending on the electric and magnetic fields respectively. It is not obvious that these are correctly identified as the electric and magnetic dipole coefficients, because the electric field may induce a charge density with non-zero angular momentum. We will now show that the first term is due solely to the electric dipole. Using the continuity equation and an integration by parts, we find

$$
\operatorname{Re}\left(\boldsymbol{j} \mid \boldsymbol{E}_{\mathrm{ext}}\right)=\frac{\omega}{e} \operatorname{Im}\left(\rho \mid \phi_{\mathrm{ext}}\right)=\frac{\omega}{e} \operatorname{Im}\left[\boldsymbol{d} \cdot \boldsymbol{E}_{\mathrm{ext}}\right] .
$$

The electrically induced absorption therefore depends only upon the induced dipole moment, and is independent of the magnetic moment induced by the electric field.

\section{Equivalence with perturbation theory}

In this section, we concentrate on the electric absorption. We describe an alternative approach to calculating the absorption coefficient, which was used in [7, 11-13], and show that it is equivalent to the first term in (2.37) provided the polarization operator $\hat{\Pi}(\omega)$ is related in a simple way to a propagator $\hat{P}(\omega)$. This relation will be established in the appendix.

We will consider the action of the effective potential $\phi_{\text {eff }}(\boldsymbol{r}, t)$ on the electrons. We may use either quantum mechanical or classical perturbation theory. We will describe the quantum mechanical approach, and will use semiclassical approximations: a classical theory in which quantum mechanics only enters in choosing the Fermi-Dirac distribution for the initial distribution of electrons gives identical results. Conceptually, the simplest method for calculating the absorption is to use the Fermi golden rule. This is expressed in terms of matrix elements $\phi_{n m}$ of the perturbation in the basis $\left|\psi_{i}\right\rangle$ formed by the eigenstates of the single-particle effective Hamiltonian, $\hat{H}_{\text {eff }}$ :

$$
\begin{aligned}
& \hat{H}_{\text {eff }}\left|\psi_{i}\right\rangle=E_{i}\left|\psi_{i}\right\rangle \\
& \phi_{i j}=\left\langle\psi_{i}|\hat{\phi}| \psi_{j}\right\rangle \quad \hat{\phi}=\phi_{\text {eff }}(\hat{\boldsymbol{r}}) .
\end{aligned}
$$


The Fermi golden rule states that the rate of transition from an initially occupied state to a quasi-continuum of final states, with density of states $n$ and with energy differing by $\hbar \omega$ from the original state, is given by

$$
R=\frac{\pi n e^{2}}{2 \hbar}\left\langle\left|\phi_{i j}\right|^{2}\right\rangle
$$

where the angle brackets denote an average over matrix elements $\left\langle\psi_{i}\left|\hat{\phi}_{\text {eff }}\right| \psi_{j}\right\rangle$. We will consider the case where both the temperature and the photon energy are small compared to other energy scales in the problem; generalizations are straightforward. Absorption of energy occurs due to the excitation of electrons in occupied states below the Fermi level to empty states above the Fermi level. The number of states which can be excited is $\sim n \hbar \omega$, and the energy absorbed in each transition is $\hbar \omega$ : the total rate of absorption of energy is given by multiplying these factors by the transition rate $R$, giving

$$
\frac{\overline{\mathrm{d} E}}{\mathrm{~d} t}=\frac{1}{2} \pi \hbar n^{2} \omega^{2}\left\langle\left|\phi_{i j}\right|^{2}\right\rangle .
$$

Both (2.37) and (3.3) are quadratic functions of $\phi_{\text {eff }}$, but it is not immediately clear how they can be related. We will now discuss why they are equivalent.

The mean square matrix element can be estimated from the correlation function $C_{\phi \phi}(t)$ of the effective potential [16]:

$$
\begin{aligned}
& \left\langle\left|\phi_{n m}\right|^{2}\right\rangle=\frac{1}{\pi \hbar n} \operatorname{Re} \int_{0}^{\infty} \mathrm{d} t \mathrm{e}^{\mathrm{i} \omega t} C_{\phi \phi}(t) \\
& C_{\phi \phi}(t)=\frac{1}{V} \int \mathrm{d} \boldsymbol{r} \int \mathrm{d} \boldsymbol{r}^{\prime} P\left(\boldsymbol{r}, \boldsymbol{r}^{\prime} ; t\right) \phi(\boldsymbol{r}) \phi\left(\boldsymbol{r}^{\prime}\right) \equiv(\phi|\hat{P}(t)| \phi)
\end{aligned}
$$

where $P\left(\boldsymbol{r}, \boldsymbol{r}^{\prime} ; t\right)$ is a propagator which gives the probability of reaching $\boldsymbol{r}^{\prime}$ from $\boldsymbol{r}$ in time $t$ :

$$
P\left(\boldsymbol{r}, \boldsymbol{r}^{\prime} ; t\right)=\theta(t)\left\langle\delta\left(\boldsymbol{r}_{t}(\boldsymbol{r}, \boldsymbol{p})-\boldsymbol{r}^{\prime}\right)\right\rangle .
$$

(The averaging is defined in the appendix.) The operator $\hat{P}(t)$ is defined by analogy with (2.22). Introducing the Fourier transform $\hat{P}(\omega)$ of the propagator, we obtain

$$
\left\langle\overline{\frac{\mathrm{d} E}{\mathrm{~d} t}}\right\rangle=\frac{1}{2} \nu \omega^{2} \operatorname{Re}\left(\phi_{\mathrm{eff}}|\hat{P}(\omega)| \phi_{\text {eff }}\right)
$$

where $v$ is the density of states per unit volume, $v=n / V$, which is assumed to be independent of position because the potential $V_{\text {eff }}$ appearing in (2.14) is constant within the conducting particle. In the appendix it will be shown that there is a general relation between the propagator and the polarizability operator:

$$
\hat{\Pi}(\omega)=e v[\hat{I}+\mathrm{i} \omega \hat{P}(\omega)] .
$$

Substituting this into (2.37) reproduces (3.7), thus establishing its equivalence to (3.3). A relation of this form has been given by Kirzhnitz et al [17]. We present a detailed derivation in the appendix, based on Liouville's equation.

\section{Simple forms for the polarizability}

In general, the operators $\hat{P}(\omega), \hat{\Pi}(\omega)$ and $\widehat{\Sigma}(\omega)$ are non-local operators. In various regimes simple approximations for these operators can be used. 


\subsection{Spatially homogeneous, ballistic system}

For a spatially homogeneous system, $\Pi\left(\boldsymbol{r}, \boldsymbol{r}^{\prime} ; \omega\right)$ is a function of $\boldsymbol{r}-\boldsymbol{r}^{\prime}$, and is conveniently represented by its Fourier transform, $\Pi(\boldsymbol{q}, \omega)$ : in $d$ dimensions,

$$
\left.\hat{\Pi}(\omega)=\frac{V}{(2 \pi)^{d}} \int \mathrm{d} \boldsymbol{q} \mid \chi_{\boldsymbol{q}}\right) \Pi(\boldsymbol{q}, \omega)\left(\chi_{\boldsymbol{q}} \mid \quad\left(\boldsymbol{r} \mid \chi_{\boldsymbol{q}}\right)=\frac{1}{\sqrt{V}} \mathrm{e}^{\mathrm{i} \boldsymbol{q} \cdot \boldsymbol{r}}\right.
$$

where $V$ is the volume of the system. In the case where the electron motion is ballistic, the propagator is, for $d=3$,

$$
\begin{aligned}
& P\left(\boldsymbol{r}, \boldsymbol{r}^{\prime} ; t\right)=\frac{1}{4 \pi R^{2}} \delta\left(R-v_{\mathrm{F}} t\right) \quad R=\left|\boldsymbol{r}-\boldsymbol{r}^{\prime}\right| \\
& P\left(\boldsymbol{r}, \boldsymbol{r}^{\prime} ; \omega\right)=\frac{1}{4 \pi v_{\mathrm{F}} R^{2}} \mathrm{e}^{-\mathrm{i} \omega R / v_{\mathrm{F}}}
\end{aligned}
$$

and the Fourier transform representation of the polarizability is

$$
\Pi(q, \omega)=v e\left(1-\frac{1}{2 \lambda} \log \left|\frac{\lambda+1}{\lambda-1}\right|+\mathrm{i} \theta(\lambda-1) \frac{\pi}{2 \lambda}\right) \quad \lambda=\left|\frac{q v_{\mathrm{F}}}{\omega}\right|
$$

which is equivalent to equations $(12.48 a),(12.48 b)$ in [5]. In two dimensions, $\Pi(q, \omega)$ is given by

$$
\Pi(q, \omega)=e v \begin{cases}1+\mathrm{i}\left(\lambda^{2}-1\right)^{-1 / 2} & \text { for }|\lambda|>1 \\ 1-\left(1-\lambda^{2}\right)^{-1 / 2} & \text { for }|\lambda|<1\end{cases}
$$

\subsection{Low- and high-frequency limits}

In the low-frequency limit, it is immediately clear from (3.8) that the induced charge density is $\rho(\boldsymbol{r})=\operatorname{ve\phi }(\boldsymbol{r})$, so

$$
\Pi\left(\boldsymbol{r}, \boldsymbol{r}^{\prime} ; \omega\right) \sim \operatorname{ve\delta }\left(\boldsymbol{r}-\boldsymbol{r}^{\prime}\right) \quad \omega \ll \omega_{\mathrm{c}} .
$$

For sufficiently high frequencies, and sufficiently far from the surface of the particle, the conductivity is local, with value $\sigma(\omega)$ :

$$
\Sigma_{i j}\left(\boldsymbol{r}, \boldsymbol{r}^{\prime} ; \omega\right)=\delta_{i j} \delta\left(\boldsymbol{r}-\boldsymbol{r}^{\prime}\right) \sigma(\omega)
$$

and the bulk conductivity $\sigma(\omega)$ may, in the case of diffusive electron motion, be approximated by the Drude formula

$$
\sigma(\omega)=\frac{v e^{2} D}{1+\mathrm{i} \omega \tau} \quad \tau=\frac{m}{N} \frac{\partial N}{\partial E} D
$$

where $D$ is the diffusion constant, $m$ is the electron effective mass and $N$ is the density of electrons. In the case of ballistic electron motion, the bulk conductivity is determined purely by the inertia of the electrons, and is non-dissipative:

$$
\sigma(\omega)=\frac{N e^{2}}{i m \omega}
$$

When the non-local conductivity can be approximated by (4.6), the non-local polarizability takes the simple form

$$
\Pi\left(\boldsymbol{r}, \boldsymbol{r}^{\prime} ; \omega\right)=\frac{\mathrm{i} \sigma(\omega)}{e \omega} \nabla^{2} \delta\left(\boldsymbol{r}-\boldsymbol{r}^{\prime}\right)
$$

This approximation is expected to be valid when $\omega \gg \omega_{\mathrm{c}}$, and when both $r$ and $\boldsymbol{r}^{\prime}$ are much greater than a distance $\Lambda$ from the surface: in the ballistic case $\Lambda=v_{\mathrm{F}} / \omega$, and in the diffusive case $\Lambda=\sqrt{D / \omega}$. The same conclusion can also be reached by considering the expressions (4.3), (4.4) in the limit $\lambda \rightarrow 0$ : for $d=3$ we find that $\Pi(q, \omega) \sim \frac{1}{3} v e \lambda^{2}=v e v_{\mathrm{F}}^{2} q^{2} / 3 \omega^{2}$, which is equivalent to the Fourier transform of (4.9) when the conductivity is given by (4.8). 


\subsection{Polarizability close to a surface}

Next we consider the polarization charge close to the surface of the particle. Here we are concerned with the high-frequency case, $\omega \gg \omega_{\mathrm{c}}$. In the low-frequency case (4.4) gives an adequate approximation, but our discussion of the high-frequency case assumed that the conductivity could be approximated as that of a homogeneous system. Another reason for discussing the surface separately is that, in our semiclassical theory, we expect it to be possible for the polarization charge density to have a singularity there.

We may assume that for $\omega \gg \omega_{\mathrm{c}}$ the polarizability operator is short ranged. A smooth surface may therefore be approximated locally by a flat surface, $z=0$ in some local Cartesian coordinates. The polarizability is given by (3.8), and we approximate the propagator from $\boldsymbol{r}^{\prime}=\left(x^{\prime}, y^{\prime}, z^{\prime}\right)$ to $\boldsymbol{r}=(x, y, z)$ by the sum of a direct contribution and a contribution originating from an image source at $r_{\mathrm{im}}^{\prime}=\left(x^{\prime}, y^{\prime},-z^{\prime}\right)$, so

$$
\rho(\boldsymbol{r})=v e\left[\phi(r)+\mathrm{i} \omega \int \mathrm{d} \boldsymbol{r}^{\prime}\left[P\left(\boldsymbol{r}, \boldsymbol{r}^{\prime} ; \omega\right)+P\left(\boldsymbol{r}, \boldsymbol{r}_{\mathrm{im}}^{\prime} ; \omega\right)\right] \phi\left(\boldsymbol{r}^{\prime}\right)\right] .
$$

The charge density is concentrated in a narrow layer at the surface, and may typically be approximated by writing

$$
\rho(\boldsymbol{r})=\rho_{s}(z) K(\boldsymbol{S})
$$

where $S$ labels points on the surface and $z$ is a coordinate normal to the surface. In this case, the potential in the neighbourhood of the surface is of the form $\phi(r)=\phi_{S}(z) K(\boldsymbol{S})$, where $\phi_{s}(z)$ satisfies

$\left.\rho_{s}(z)=v e\left[\phi_{s}(z)+\frac{1}{\Lambda} \int_{0}^{\infty} \mathrm{d} z^{\prime}\left[G\left(\left(z-z^{\prime}\right) / \Lambda\right)\right)+G\left(\left(z+z^{\prime}\right) / \Lambda\right)\right] \phi_{s}\left(z^{\prime}\right)\right]$

where $\Lambda=v_{\mathrm{F}} / \omega$, and the function $G(x)$ is easily related to the Fourier transform of $\Pi(q, \omega)$.

\subsection{Diffusive electron motion}

In the diffusive case, it is possible to write a useful eigenfunction expansion for the linear response functions: for $t>0$ the propagator $P\left(\boldsymbol{r}, \boldsymbol{r}^{\prime} ; t\right)$ satisfies the diffusion equation $\partial_{t} P-D \nabla^{2} P=\delta\left(r-r^{\prime}\right) \delta(t)$, or $\left[\mathrm{i} \omega-D \nabla^{2}\right] P\left(\boldsymbol{r}, \boldsymbol{r}^{\prime} ; \omega\right)=\delta\left(\boldsymbol{r}-\boldsymbol{r}^{\prime}\right)$, and satisfies the Neumann boundary condition. It can be expressed in terms of a set of eigenfunctions $\chi_{n}(r)$ of the Helmholtz equation $\left(\nabla^{2}+k_{n}^{2}\right) \chi_{n}(r)=0$, satisfying the same boundary condition: $\hat{\boldsymbol{n}} \cdot \nabla \chi_{n}=0$, where $\hat{\boldsymbol{n}}$ is a normal vector on the surface of the particle. The propagator can then be written

$$
\left.\hat{P}(\omega)=\sum_{n} \frac{1}{\mathrm{i} \omega-D k_{n}^{2}} \mid \chi_{n}\right)\left(\chi_{n} \mid .\right.
$$

Expansions for other linear response functions are easily obtained in the same form. For example, if the density of states per unit volume $v$ is independent of $\boldsymbol{r}$, equation (3.8) implies that the polarizability can be written in this form, with the coefficient of the operator $\left.\mid \chi_{n}\right)\left(\chi_{n} \mid\right.$ given by $v e D k_{n}^{2} /\left(D k_{n}^{2}-\mathrm{i} \omega\right)$.

\section{The self-consistent field}

\subsection{Approximate equations for the self-consistent fields}

Here we discuss how the solution of the self-consistent equations can be greatly simplified by the use of 'semiclassical' approximations. We consider the electric dipole absorption first. 
Calculation of the electric dipole absorption coefficient via either (2.37) or (3.7) requires the self-consistent field $\phi_{\text {eff }}(\boldsymbol{r}, \omega)$, which is given by equation (2.30):

$$
\left.\left.\mid \phi_{\mathrm{ext}}\right)=[\hat{I}-\hat{U} \hat{\Pi}(\omega)] \mid \phi_{\mathrm{eff}}\right) .
$$

Formally, solution of this equation requires calculation of the inverse of $\hat{I}-\hat{U} \hat{\Pi}(\omega)$ : this could be done explicitly in a numerical calculation by expanding in a suitable basis set. We will aim instead for an approximate analytic solution. For frequencies small compared to the plasma frequency $\omega_{\mathrm{p}}$, the external electric field is 'screened' by polarization charges, so the internal field is much smaller than the externally applied field. The key physical intuition is that the external electric field is almost exactly cancelled by the electric field due to the induced charge density $\rho(\boldsymbol{r})$. Let $\rho_{\mathrm{cl}}(\boldsymbol{r})$ be the charge density induced on the particle by a static external field, according to classical electrodynamics: this charge density gives an induced electric field which precisely cancels the externally applied field inside the particle. For frequencies small compared to the plasma frequency, the induced charge density is well approximated by $\rho_{\mathrm{cl}}(\boldsymbol{r})$ : we will assume that

$$
\left.\mid \rho)=\mid \rho_{\mathrm{cl}}\right)+\mathrm{O}\left(\omega / \omega_{\mathrm{p}}\right)+\mathrm{O}\left(a_{0} / a\right)
$$

where $a$ is the characteristic dimension of the particle and $a_{0}$ is the Bohr radius. The classical charge distribution formally satisfies an equation analogous to (5.1), in which the term representing the internal field $\left.\mid \phi_{\text {eff }}\right)$ is set equal to zero:

$$
\left.\mid \phi_{\text {ext }}\right)+\hat{U}\left(\rho_{\mathrm{cl}}\right)=0 \text {. }
$$

The general solution of this equation to determine $\left.\mid \rho_{\mathrm{cl}}\right)$ is very difficult, but textbooks such as [6] or [18] contain solutions for conductors with a variety of simple geometries. We will assume that the surface charge density $\left.\mid \rho_{\mathrm{cl}}\right)$ is available. We will denote our approximation to the effective potential $\left.\mid \phi_{\text {eff }}\right)$ by $\left.\mid \phi\right)$ : it is the potential which generates the polarization charge $\left.\mid \rho_{\mathrm{cl}}\right)$, and is given by

$$
\left.\left.\mid \rho_{\mathrm{cl}}\right)=\hat{\Pi}(\omega) \mid \phi\right)
$$

or equivalently by $\left.\left.\mid \phi_{\text {ext }}\right)+\hat{U} \hat{\Pi}(\omega) \mid \phi\right)=0$. Comparing with (5.1), it is clear that this solution $\mid \phi)$ is a good approximation to $\left.\mid \phi_{\text {eff }}\right)$ provided $\|\hat{U} \hat{\Pi}(\omega)\| \gg 1$, where $\|\hat{X}\|$ is an appropriate norm of the operator $\hat{X}$. To estimate this norm, we consider the effect of an arbitrary potential $\phi$ : when $\omega \ll \omega_{\mathrm{c}}$, the induced charge density is $\hat{\Pi} \phi=e v \phi$, and for a particle of characteristic dimension $a$ in $d$ dimensions, the induced charge may be approximated by a dipole formed by charges of magnitude $Q \sim \rho a^{d}$, with separation $a$ : this results in an electrical potential of magnitude $\phi^{\prime} \sim e Q /\left(\epsilon_{0} a\right)$. In three-dimensional particles with ballistic electron motion, this leads to the following estimate for $\|\hat{U} \hat{\Pi}\| \sim \phi^{\prime} / \phi$ :

$$
\|\hat{U} \hat{\Pi}\| \sim \frac{\omega_{\mathrm{p}}^{2}}{\omega_{\mathrm{c}}^{2}}
$$

where $\omega_{\mathrm{p}}$ is the three-dimensional bulk plasma frequency, $\omega_{\mathrm{p}}=\left[N e^{2} /\left(\epsilon_{0} m\right)\right]^{1 / 2}, N$ being the electron density. For frequencies $\omega \gg \omega_{\mathrm{c}}$, a similar argument gives

$$
\left\|\hat{U} \hat{\Pi}\left(\omega \gg \omega_{\mathrm{c}}\right)\right\| \sim \frac{\omega_{\mathrm{p}}^{2}}{\omega \omega_{\mathrm{c}}} .
$$

The ordering frequency scales given in (1.1) imply that (5.5) and (5.6) are indeed large.

We can, in principle, determine improved approximations to the exact solution of (5.1) from the solution of (5.4):

$$
\left.\left.\left.\left.\mid \phi_{\mathrm{eff}}\right)=-[\hat{I}-\hat{U} \hat{\Pi}(\omega)]^{-1} \hat{U} \hat{\Pi}(\omega) \mid \phi\right)=\mid \phi\right)-[\hat{I}-\hat{U} \hat{\Pi}(\omega)]^{-1} \mid \phi\right) .
$$


Equations (5.5) and (5.6) show that the correction term in (5.7) is small. Having shown that $\left.\left.\mid \phi_{\text {eff }}\right) \sim \mid \phi\right)$, we discuss how to estimate the solution $\left.\mid \phi\right)$ of (5.4) in sections 5.2 and 5.3.

Finally we consider the semiclassical solution for the magnetically induced field, $\boldsymbol{E}_{\text {ind }}$, which satisfies (2.29):

$$
\left.\boldsymbol{\nabla} \cdot\left[\hat{I}-\frac{\mathrm{i}}{\omega \epsilon_{0}} \widehat{\Sigma}(\omega)\right] \mid \boldsymbol{E}_{\text {ind }}\right)=0 .
$$

At low frequencies we can estimate the conductivity by $\Sigma \sim N e^{2} / m \omega_{\mathrm{s}}$ where $\omega_{\mathrm{s}}$ is the scattering frequency; at high frequencies $\omega_{\mathrm{s}}$ is replaced by the frequency $\omega$. In the lowfrequency limit we therefore estimate

$$
\frac{1}{\epsilon_{0} \omega}\|\widehat{\Sigma}(\omega)\| \sim \frac{\omega_{\mathrm{p}}^{2}}{\omega \omega_{\mathrm{s}}} .
$$

In the frequency ranges that we are concerned with, the term involving the identity operator in (5.8) is therefore negligible. We can therefore find an approximate solution to (5.8) by requiring that the induced charge density is zero, i.e.

$$
\left.\boldsymbol{\nabla} \cdot \widehat{\Sigma}(\omega) \mid \boldsymbol{E}_{\text {ind }}\right)=0 .
$$

This justifies the neglect of a cross-term in (2.1) with an electric dipole induced by the magnetic field. A solution to (5.10) may be determined by choosing an initial approximation $\boldsymbol{E}_{\text {ind }}^{\prime}$ which satisfies $\boldsymbol{\nabla} \wedge \boldsymbol{E}_{\text {ind }}^{\prime}=\mathrm{i} \omega \boldsymbol{B}_{\text {ext }}$. A polarization charge $\rho_{\text {ind }}^{\prime}$ would be generated from this field. An additional field which is the gradient of a potential $\chi(r)$ is added, such that $\boldsymbol{\nabla} \chi=e\left(\boldsymbol{E}_{\text {ind }}^{\prime}-\boldsymbol{E}_{\text {ind }}\right)$. The condition upon $\chi$ for (5.10) to be satisfied is

$$
\left.\left.\mid \rho_{\text {ind }}^{\prime}\right)=\hat{\Pi}(\omega) \mid \chi\right) .
$$

This equation is analogous to (5.4).

\subsection{The ballistic case}

We will discuss approximate solutions of (5.4) valid in the limits $\omega \ll \omega_{\mathrm{c}}$ and $\omega \gg \omega_{\mathrm{c}}$. The first of these represents the static potential required to hold the classical charge distribution in place in the zero-frequency limit: it will be written $\left.\mid \phi_{\text {stat }}\right)$, and its form is immediately apparent from (3.8):

$$
\left.\left.\mid \phi_{\text {stat }}\right)=\frac{1}{e v} \mid \rho_{\mathrm{cl}}\right) .
$$

This is simply a linearized Thomas-Fermi approximation [19]. The semiclassical approximations underlying this expression assume that the potentials are slowly varying on the scale of the Fermi wavelength.

In the limit $\omega \gg \omega_{\mathrm{c}}$, we found (equation (4.9)) that the polarizability may be approximated by $\hat{\Pi} \sim(\mathrm{i} \sigma(\omega) / e \omega) \nabla_{r}^{2} \delta\left(\boldsymbol{r}-\boldsymbol{r}^{\prime}\right)$, for $\boldsymbol{r}$ and $\boldsymbol{r}^{\prime}$ not too close to the surface. For points not too close to the surface, or to points where the charge density is non-analytic, we can approximate the solution of (5.4) by a 'dynamic' potential, which is of the form $\left.\left.\mid \phi_{\mathrm{dyn}}\right)=\lambda \mid \psi\right)$, where $\nabla^{2} \psi(\boldsymbol{r})=\rho_{\mathrm{cl}}(\boldsymbol{r})$, and $\hat{\boldsymbol{n}} \cdot \nabla \psi(\boldsymbol{r})=0$. Substituting these forms into (5.4) we find that $\lambda=\mathrm{i} \omega e / \sigma(\omega)$ :

$$
\left.\left.\mid \phi_{\mathrm{dyn}}\right)=-\frac{\mathrm{i} \omega e}{\sigma(\omega)}|\psi\rangle \quad \nabla^{2}|\psi\rangle=\mid \rho_{\mathrm{cl}}\right) .
$$

An interpretation of the dynamic potential is that it moves the polarization charge into place.

Close to the surface, equation (5.13) is not necessarily a good approximation to the effective potential. One reason is that the approximations underlying (4.9) fail, and the polarization must 
be described by (4.9) or (4.12). Another reason is that the charge density $\left.\mid \rho_{\mathrm{cl}}\right)$ has a singularity there. In the notation of (4.11), the projected charge density $\rho_{s}(z)$ is concentrated on the surface in the three-dimensional case [11] so $\rho_{s}(z) \sim \delta(z)$, and in the two-dimensional case it diverges on the surface, such that $\rho_{s}(z) \sim z^{-1 / 2}$ for $z>0$ [7]. The form of (4.12) indicates that the potential $\phi_{s}(z)$ also has the same type of singularity as the charge density at the surface.

To summarize, the following picture emerges. For low frequencies, $\omega \ll \omega_{\mathrm{c}}$, the potential is approximately $\phi_{\text {stat }}(\boldsymbol{r})$. At high frequencies, $\omega \gg \omega_{\mathrm{c}}$, the potential is well approximated by $\phi_{\text {dyn }}(r)$ within the interior of the particle. In the vicinity of the surface, the potential has a dominant divergent contribution, which is well approximated by $\phi_{\text {stat }}(\boldsymbol{r})$.

\subsection{The diffusive case}

In the diffusive case, equation (5.4) can be solved exactly, using the representation of the propagator in the form (4.13). Expanding the potential $\mid \phi$ ) in terms of the functions $\mid \chi_{n}$ ) leads to the expression

$$
\left.\left.\left.\mid \phi)=\frac{1}{e v} \sum_{n}\left(\chi_{n} \mid \rho_{\mathrm{cl}}\right)\left[1-\frac{\mathrm{i} \omega}{D k_{n}^{2}}\right] \mid \chi_{n}\right)=\mid \phi_{\text {stat }}\right)+\mid \phi_{\text {dyn }}\right) .
$$

Note that in the diffusive case the potential is precisely equal to the sum of the static and dynamic contributions [13].

\section{Discussion: calculation of the absorption coefficient}

Once an adequate approximation for the effective potential has been obtained, the electric absorption coefficient is obtained from (3.7): the absorption coefficient is proportional to $\omega^{2}(\phi|\hat{P}(\omega)| \phi)$. Previous papers [7, 11-14] have discussed methods for the evaluation of the absorption coefficient using equation (3.7) in some detail, for specific cases. This paper has presented a general approach to the determining of the effective potential, and some remarks on applying this to calculating the absorption coefficient are appropriate.

When the electron motion is diffusive, the absorption is very easily evaluated using (5.14) and (4.13). It is found that the coefficient is proportional to $\omega^{2}$, and that (at least within the framework of the approximations used in section 5) the frequency scale $\omega_{c}$ plays no role. The absorption coefficient can be shown to be exactly equal to the classical value in this case $[12,13]$.

The case of ballistic electron motion is more difficult. It might be expected that $C_{\phi \phi}(\omega)=(\phi|\hat{P}(\omega)| \phi)$ approaches a non-zero limit as $\omega \rightarrow 0$, implying that the absorption coefficient is proportional to $\omega^{2}$ for low frequencies. This expectation is correct for cases where the electron motion is ergodic (the most important cases being diffusive electron motion, and the ballistic case with a rough surface). In the case of integrable electron motion, which can be realized experimentally if the surface appears smooth on the scale of the Fermi wavelength, $C_{\phi \phi}(\omega)$ typically approaches zero as $\omega \rightarrow 0$ in a non-analytic fashion. In the important special case of particles with circular symmetry, $C_{\phi \phi}(\omega)$ is zero for $\omega<\omega_{\mathrm{c}}$, where $\omega_{\mathrm{c}}$ is the frequency of a glancing circular orbit. The electron dynamics therefore plays an important role in determining the low-frequency absorption.

The high-frequency absorption, by contrast, is determined by the nature of the singularities of the function $f(t)=\phi\left(\boldsymbol{r}_{t}\right)$, which can result from singularities in the motion $\boldsymbol{r}(t)$, or in the potential $\phi(r)$. We will discuss the ballistic case. The dominant contribution comes from the singularities of $\phi(r)$ in the neighbourhood of the surface. The absorption coefficient was calculated in [11] and [7] for the three-dimensional and two-dimensional cases respectively, 
assuming that the effective potential is equal to $\phi_{\text {stat }}(r)$. In three dimensions, this potential is a delta function singularity concentrated on the surface, and in two dimensions it diverges as $z^{-1 / 2}$, where $z$ is the normal distance from the surface. These forms for the potential imply that $(\phi|\hat{P}(\omega)| \phi) \sim \omega^{0}$ in three dimensions and $\sim \omega^{-1}$ in two dimensions. In [7] and [11] it was predicted that in the case of particles with circular symmetry, the absorption coefficient shows a sequence of resonances superposed on a regular contribution increasing as $\omega^{2}$ and $\omega$ for three and two dimensions respectively.

The more sophisticated approach introduced in this paper indicates that the potential $|\phi\rangle$ should satisfy (5.4), whereas the potential used in $[11,7,20]$ was simply the static potential, satisfying $\left.\left.\mid \rho_{\mathrm{cl}}\right)=\hat{\Pi}(0) \mid \phi_{\text {stat }}\right)$. It is necessary to consider the extent to which this refinement will change the results. In section 5.2 we argued that the singularities of the effective potential at the surface are the same as those of the 'static' potential. We can therefore hypothesize that the more refined theory would make a quantitative rather than qualitative difference to the results. This issue will be addressed in a subsequent paper, which will consider the inversion of (4.12) to determine the potential $\phi_{s}(z)$ from the charge density $\rho_{s}(z)$, and its use to estimate the high-frequency absorption coefficient [15].

\section{Acknowledgments}

BM would like to acknowledge support of the SFB393 (project C5); the work of MW was supported by a research grant from the EPSRC, reference GR/L02302.

\section{Appendix}

We will now relate the polarizability operator $\Pi\left(\boldsymbol{r}, \boldsymbol{r}^{\prime} ; \omega\right)$ to the probability propagator $P\left(r, r^{\prime} ; t\right)$, which is the probability that an electron, released at $r^{\prime}$ with energy equal to the Fermi energy $E_{\mathrm{F}}$, will be at position $r$ after time $t$. The discussion will be classical; a quantum mechanical derivation proceeds along similar lines.

Let the phase-space distribution be $f(\boldsymbol{r}, \boldsymbol{p} ; t)$ : this will, when convenient, be written $f(\alpha, t)$ where $\alpha=(\boldsymbol{r}, \boldsymbol{p})$. The Hamiltonian will be assumed to be of the form

$$
H(\alpha, t)=H_{0}(\alpha)+X(t) H_{1}(\alpha)
$$

where we will be interested in the case where $H_{0}=p^{2} / 2 m+V(r)$ and $H_{1}=\phi(r)$. The perturbation parameter $X(t)$ is assumed to be small, so that $f(\alpha, t)$ may be expanded as a series in $X(t)$ : we will be interested in the expansion as far as the first-order term:

$$
f(\alpha, t)=f_{0}(\alpha)+\int_{-\infty}^{t} \mathrm{~d} t^{\prime} X\left(t^{\prime}\right) g\left(\alpha, t, t^{\prime}\right)+\mathrm{O}\left(X^{2}\right) .
$$

Substituting into the Liouville equation, $\partial_{t} f=\{H, f\}$, it is found that $f_{0}$ is a function of the unperturbed Hamiltonian $H_{0}(\alpha)$, and that the kernel $g\left(\alpha, t, t^{\prime}\right)$ of the first-order term satisfies

$$
X(t)\left[g(\alpha, t, t)-\left\{H_{1}, f_{0}\right\}_{\alpha}\right]+\int_{-\infty}^{t} \mathrm{~d} t^{\prime} X\left(t^{\prime}\right)\left[\partial_{t} g-\left\{H_{0}, g\right\}\right]_{\alpha, t, t^{\prime}}=0
$$

which is valid for all $X(t)$. The first term implies that

$$
g(\alpha, t, t)=g(\alpha)=\left\{H_{1}, f_{0}\right\}_{\alpha}=\left\{H_{1}, H_{0}\right\}_{\alpha} \frac{\partial f_{0}}{\partial E}\left(H_{0}(\alpha)\right) .
$$

The second term implies that $\mathrm{d} g / \mathrm{d} t=0$, where $\mathrm{d} / \mathrm{d} t$ is the total time derivative along a trajectory, so

$$
g\left(\alpha, t, t^{\prime}\right)=g\left(\alpha, t-t^{\prime}\right)=\frac{\partial f_{0}}{\partial E}\left(H_{0}(\alpha)\right) \frac{\mathrm{d} H_{1}}{\mathrm{~d} t}\left(\alpha_{t-t^{\prime}}(\alpha)\right) .
$$


The required approximation is then

$$
f(\alpha, t)=f_{0}\left(H_{0}(\alpha)\right)+\frac{\partial f_{0}}{\partial E}\left(H_{0}(\alpha)\right) \int_{-\infty}^{t} \mathrm{~d} t^{\prime} X\left(t^{\prime}\right) \frac{\mathrm{d} H_{1}}{\mathrm{~d} t}\left(\alpha_{t-t^{\prime}}\right) .
$$

We will use an alternative form, obtained by integration by parts:

$f(\alpha, t)=f_{0}\left(H_{0}(\alpha)\right)+X(t) \frac{\partial f_{0}}{\partial E}\left(H_{0}(\alpha)\right) H_{1}(\alpha)-\frac{\partial f_{0}}{\partial E}\left(H_{0}(\alpha)\right) \int_{-\infty}^{t} \mathrm{~d} t^{\prime} \dot{X}\left(t^{\prime}\right) H_{1}\left(\alpha_{t-t^{\prime}}(\alpha)\right)$.

We will assume that the integral converges. For ergodic systems this requires that the microcanonical average of $H_{1}(\alpha)$ vanishes. The density of available states in phase space is $(2 \pi \hbar)^{-d}$, where $d$ is the number of degrees of freedom. For a system of fermions, the appropriate density function is $f_{0}(\alpha, X)=\Theta\left(H(\alpha, X)-E_{\mathrm{F}}\right) /(2 \pi \hbar)^{d}$, where $\Theta(x)$ is the Fermi-Dirac distribution, which can be approximated by a downward step function when the temperature is small compared to the Fermi temperature. Now the charge density of electrons is

$$
\rho(\boldsymbol{r}, t)=-e \int \mathrm{d} \boldsymbol{p} f(\boldsymbol{r}, \boldsymbol{p} ; t) .
$$

The number density of electrons $N\left(E_{\mathrm{F}}, \boldsymbol{r}\right)$ and the density of states per unit volume at the Fermi surface $v\left(E_{\mathrm{F}}, \boldsymbol{r}\right)$ are

$$
N\left(E_{\mathrm{F}}, \boldsymbol{r}\right)=\frac{1}{(2 \pi \hbar)^{d}} \int \mathrm{d} \boldsymbol{p} \Theta\left(H_{0}(\boldsymbol{r}, \boldsymbol{p})-E_{\mathrm{F}}\right) \quad v\left(E_{\mathrm{F}}, \boldsymbol{r}\right)=\frac{\partial}{\partial E_{\mathrm{F}}} N\left(E_{\mathrm{F}}, \boldsymbol{r}\right)
$$

respectively. Also, the local average of any quantity $A(r, p)$ for electrons at the Fermi surface is defined as

$$
\langle A\rangle_{E_{\mathrm{F}}, \boldsymbol{r}}=\frac{1}{(2 \pi \hbar)^{d} v\left(E_{\mathrm{F}}, \boldsymbol{r}\right)} \int \mathrm{d} \boldsymbol{p} A(\boldsymbol{r}, \boldsymbol{p}) \delta\left(H_{0}(\boldsymbol{r}, \boldsymbol{p})-E_{\mathrm{F}}\right) .
$$

From (A.7) and the definition (A.8), we have

$$
\begin{aligned}
& \rho(\boldsymbol{r}, t) \sim-e N\left(E_{\mathrm{F}}, \boldsymbol{r}\right)+e X(t) v\left(E_{\mathrm{F}}, \boldsymbol{r}\right) \phi(\boldsymbol{r}) \\
& \quad-e v\left(E_{\mathrm{F}}, \boldsymbol{r}\right) \int_{-\infty}^{t} \mathrm{~d} t^{\prime} \dot{X}\left(t^{\prime}\right) \int \mathrm{d} \boldsymbol{r}^{\prime}\left\langle\delta\left[\boldsymbol{r}^{\prime}-\boldsymbol{r}_{t-t^{\prime}}(\boldsymbol{r}, \boldsymbol{p})\right]\right\rangle_{E_{\mathrm{F}}, \boldsymbol{r}} \phi\left(\boldsymbol{r}_{t^{\prime}}\right)+\mathrm{O}\left(X^{2}\right) .
\end{aligned}
$$

With the definition of the propagator (3.6) and recalling the definition of the polarization operator, equation (2.23), we find

$$
\Pi\left(\boldsymbol{r}, \boldsymbol{r}^{\prime}, t-t^{\prime}\right)=e \theta\left(t-t^{\prime}\right) v\left(E_{\mathrm{F}}, \boldsymbol{r}\right)\left[\delta\left(\boldsymbol{r}-\boldsymbol{r}^{\prime}\right) \delta\left(t-t^{\prime}\right)+\partial_{t} P\left(\boldsymbol{r}, \boldsymbol{r}^{\prime} ; t-t^{\prime}\right)\right]
$$

or alternatively, in the frequency domain,

$$
\Pi\left(\boldsymbol{r}, \boldsymbol{r}^{\prime}, \omega\right)=e v\left(E_{\mathrm{F}}, \boldsymbol{r}\right)\left[\delta\left(\boldsymbol{r}-\boldsymbol{r}^{\prime}\right)+\mathrm{i} \omega P\left(\boldsymbol{r}, \boldsymbol{r}^{\prime} ; \omega\right)\right] .
$$

We will introduce an operator $\hat{v}$, which is diagonal in the position representation, such that $(\boldsymbol{r}|\hat{v}| \phi)=v\left(E_{\mathrm{F}}, \boldsymbol{r}\right) \phi(\boldsymbol{r})$. Equation (A.13) may then be written in the form

$$
\hat{\Pi}(\omega)=e \hat{v}[\hat{I}+\mathrm{i} \omega \hat{P}(\omega)]
$$

There is also a relationship between the non-local conductivity $\widehat{\Sigma}(\omega)$ and the propagator $\hat{P}(\omega)$, which has previously been obtained by Serota and co-workers [21, 22] (with an alternative derivation given in [14]). Their derivation was specific to the case of diffusive electron motion, whereas that given above also includes the ballistic case. 


\section{References}

[1] Carr G L, Perkowitz S and Tanner D B 1985 Infrared and Millimeter Waves vol 13, ed K J Button (New York: Academic) p 169

[2] Perenboom J A A J, Wyder P and Meier F 1981 Phys. Rep. 78173

[3] Devaty R P 1989 Physica A 157262

[4] Bertsch G F and Broglia R A 1994 Oscillations in Finite Quantum Systems (Cambridge: Cambridge University Press)

[5] Fetter A L and Walecka J D 1971 Quantum Theory of Many-Particle Systems (New York: McGraw-Hill)

[6] Landau L D and Lifshitz E M 1958 Electrodynamics of Continuous Media (Landau and Lifshitz Course of Theoretical Physics 8) (Oxford: Pergamon)

[7] Wilkinson M and Austin E J 1994 J. Phys.: Condens. Matter 64153

[8] Gorkov L P and Eliashberg G M 1965 Zh. Eksp. Teor. Fiz. 481407

[9] Mehta M L 1991 Random Matrices 2nd edn (New York: Academic)

[10] Beenakker C W J and van Houten 1991 Solid State Physics vol 44 (New York: Academic) pp 1-228

[11] Austin E J and Wilkinson M 1993 J. Phys.: Condens. Matter 58461

[12] Mehlig B and Wilkinson M 1997 J. Phys.: Condens. Matter 93277

[13] Wilkinson M and Mehlig B 1998 Eur. J. Phys. B 1397

[14] Wilkinson M, Mehlig B and Walker P N 1998 J. Phys.: Condens. Matter 102739

[15] Wilkinson M 2001 J. Phys.: Condens. Matter cond-mat/0011205 preprint

[16] Wilkinson M 1987 J. Phys. A: Math. Gen. 202415

[17] Kirzhnitz D A, Lozovik Yu E and Shpatakovskaya G V 1975 Sov. Phys.-Usp. 18649

[18] Jackson J D 1999 Classical Electrodynamics (New York: Wiley)

[19] Ashcroft N W and Mermin N D 1976 Solid State Physics (Philadelphia, PA: Saunders College)

[20] Mehlig B and Richter K 1998 Phys. Rev. Lett. 801936

[21] Kane C L, Serota R A and Lee P A 1988 Phys. Rev. B 376701

[22] Serota R A, Yu J and Kim Y H 1990 Phys. Rev. B 429724 